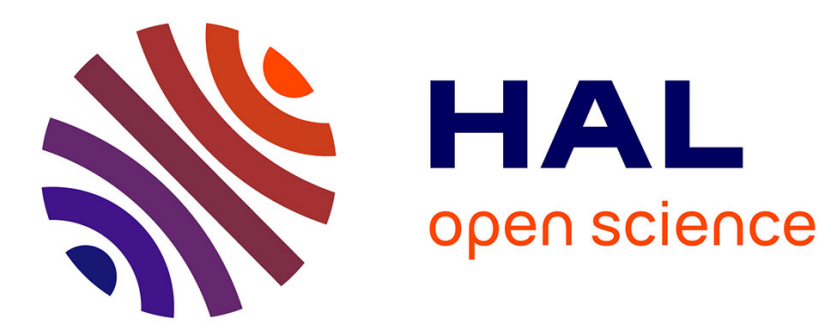

\title{
Identification of a 2-Additive Bi-Capacity by Using Mathematical Programming
}

Julien Ah-Pine, Brice Mayag, Antoine Rolland

\section{To cite this version:}

Julien Ah-Pine, Brice Mayag, Antoine Rolland. Identification of a 2-Additive Bi-Capacity by Using Mathematical Programming. Third International Conference on Algorithmic Decision Theory (ADT 2013), Nov 2013, Bruxelles, Belgium. pp.15-29, 10.1007/978-3-642-41575-3_2 . hal-01504630

\section{HAL Id: hal-01504630 \\ https://hal.science/hal-01504630}

Submitted on 10 Apr 2017

HAL is a multi-disciplinary open access archive for the deposit and dissemination of scientific research documents, whether they are published or not. The documents may come from teaching and research institutions in France or abroad, or from public or private research centers.
L'archive ouverte pluridisciplinaire HAL, est destinée au dépôt et à la diffusion de documents scientifiques de niveau recherche, publiés ou non, émanant des établissements d'enseignement et de recherche français ou étrangers, des laboratoires publics ou privés. 


\title{
Identification of a 2-additive bi-capacity by using mathematical programming
}

\author{
Julien Ah-Pine ${ }^{1}$, Brice Mayag ${ }^{2}$, and Antoine Rolland ${ }^{1}$ \\ ${ }^{1}$ ERIC Lab, University of Lyon, Bron, France \\ \{julien.ah-pine, antoine.rolland\}@univ-lyon2.fr \\ ${ }^{2}$ LAMSADE, University Paris-Dauphine, Paris, France \\ brice.mayag@dauphine.fr
}

\begin{abstract}
In some multi-criteria decision making problems, it is more convenient to express the decision maker preferences in bipolar scales. In such cases, the bipolar Choquet integral with respect to bi-capacities was introduced. In this paper, we address the problem of eliciting a bipolar Choquet integral with respect to a 2 -additive bi-capacity. We assume that we are given a set of examples with (i) their scores distribution in regard to several criteria and (ii) their overall scores. We propose two types of optimization problems that allow identifying the parameters of a 2-additive bi-capacity such that the inferred bipolar Choquet integral is consistent with the given examples as much as possible. Furthermore, since the elicitation process we study has many relationships with problems in statistical machine learning, we also present the links between our models and concepts developed in the latter field.
\end{abstract}

Keywords: 2-additive bi-capacity identification, Bipolar Choquet integral, Preference elicitation

\section{Introduction}

Multi-criteria decision making $(\mathrm{MCDM})$ aims at representing the preferences of a decision maker (DM) over a set of options (or alternatives) and in regard to several criteria. It then seeks to formalize the DM's decision process through mathematical tools in order to help him make decisions over the set of alternatives. The DM's decision process is assumed to be guided by the importance and the relationships he wants to take into account regarding the criteria. Concerning the preferences representations, one possible model is the Multi-Attribute Utility Theory (MAUT) which assumes that each attribute (or criterion) provides a utility value (or score) over the set of alternatives. Then, an aggregation function is used to combine, for each option, its scores distribution (or profile) in an overall score. The latter global utility values are then employed to make decisions. There are many types of aggregation functions to model a decision process. The Choquet integral has been proved to be a versatile tool to construct overall scores (see for example [1-4]). This aggregation function is intimately based on the concept of a capacity (or fuzzy measure). In particular, it assumes that partial utilities belong to non-negative or unipolar scales. 
Unipolar scales are not always appropriate to represent the DM's preferences (see the motivating example in [5]). In some problems, bipolar scales are more convenient. This type of scales is typically composed of a negative, a positive and a neutral part which respectively allow representing a negative, a positive and a neutral affect towards an option. To apply the Choquet integral in the case of bipolar scales, the bipolar Choquet integral (BCI) was introduced in [6] and [7]. In this paper, we particularly focus on BCI which use the concept of a bi-capacity (BC) introduced in [6] and which was further studied in $[8,9]$ and in $[10,11]$. The BCI typically requires the DM to set $3^{n}-1$ values where $n$ is the number of attributes. When $n$ exceeds some units, it is impossible for the DM to set all parameters of his decision model. In order to better cope with this combinatorial burden, the BCI with respect to (w.r.t.) a 2-additive bi-capacity (2A-BC) was introduced in the following papers $[12,10]$. The 2-additivity property implies that only the interactions between at most two criteria are taken into account in a $\mathrm{BC}$, and it enables reducing the number of parameters from $3^{n}-1$ to $2 n^{2}+1$. This has facilitated the use of this aggregation function in practice.

Even though there have been many papers studying $2 \mathrm{~A}-\mathrm{BC}$, most of them have focused on theoretical aspects. In this contribution, we study the practical problem of identifying the parameters of a $2 \mathrm{~A}-\mathrm{BC}$ on the basis of information provided by the DM. This problem is also known as preference elicitation. There are different contexts in which we can proceed to the elicitation of the preference model of a DM. In our case, we assume that the DM provides the bipolar scores for a subset of (real or fictitious) options w.r.t. all criteria of the decision problem. In addition, he provides the overall bipolar scores of the same set of alternatives. These evaluated examples constitute the only data we have at our disposal. Then, the elicitation model consists in inferring the parameters of a $2 \mathrm{~A}-\mathrm{BC}$ such that the associated BCI is consistent with the preferences given by the DM on these examples. We propose optimization models that address this kind of preference elicitation problems.

Eliciting preference models is a research topic that has been studied by many researchers (see for example $[13,14]$ ). However, the BCI w.r.t. 2A-BC has not been studied very much so far. To our knowledge, the only paper that addressed this exact problem is [15]. Yet, in the latter paper, the authors assumed an elicitation process in which the DM was asked to provide cardinal information on trinary actions. This setting is different from the one considered in this paper. Our approach is also in line with the work detailed in $[14]^{1}$ about the preference elicitation using unipolar Choquet integrals with mathematical programming. In this latter work, the authors also assume that the only information provided are examples evaluated by the DM.

The elicitation process we deal with has many relationships with the problems addressed in statistical machine learning. The interconnections between preference elicitation on the one hand and machine learning on the other hand were highlighted in [16]. There has been a growing interest for the last years about cross-fertilizing these two domains by studying how the concepts devel-

\footnotetext{
$\overline{1}$ And with the papers cited therein.
} 
oped in one field can be applied in the other one. In line with this research topic, we also discuss the links between the concepts of these two domains that our models involve.

The rest of the paper is organized as follows. We recall in section 2 some basic definitions about $\mathrm{BC}$ and the properties of $2 \mathrm{~A}-\mathrm{BC}$ by using the bipolar Möbius transform defined by [10]. We then propose in section 3 two identification methods of a $2 \mathrm{~A}-\mathrm{BC}$ using linear programming and quadratic programming. In order to illustrate our proposals, we apply the different methods to a numerical example. Next, in section 4, we underline the relationships of our approaches with concepts developed in the field of machine learning. We finally conclude this paper and sketch some future works in section 5 .

\section{Bi-capacities and bipolar Choquet integrals}

Let us denote by $N=\{1, \ldots, n\}$ a finite set of $n$ criteria and $X=X_{1} \times \cdots \times X_{n}$ the set of possible alternatives, where $X_{1}, \ldots, X_{n}$ represent the attributes. For all $i \in N$, the function $u_{i}: X_{i} \rightarrow \mathbb{R}$ is called a utility function. Given an element $x=\left(x_{1}, \ldots, x_{n}\right)$, we denote by $U(x)=\left(u_{1}\left(x_{1}\right), \ldots, u_{n}\left(x_{n}\right)\right)$, the element's profile or its scores distribution. We will often write $i j, i j k$ instead of $\{i, j\}$ and $\{i, j, k\}$ respectively.

\section{$2.1 \quad$ 2-additive bi-capacities}

Let us denote by $2^{N}:=\{S \subseteq N\}$ the set of subsets of $N$ and $3^{N}:=\{(A, B) \in$ $\left.2^{N} \times 2^{N}: A \cap B=\emptyset\right\}$ the set of couples of subsets of $N$ with an empty intersection. We define on $3^{N}$ the following relation $\sqsubseteq, \forall\left(A_{1}, A_{2}\right),\left(B_{1}, B_{2}\right) \in 3^{N}$ :

$$
\left(A_{1}, A_{2}\right) \sqsubseteq\left(B_{1}, B_{2}\right) \Leftrightarrow\left[A_{1} \subseteq B_{1} \text { and } B_{2} \subseteq A_{2}\right]
$$

Definition 1 (Bi-capacity (BC) [9], [5]) A function $\nu: 3^{N} \rightarrow \mathbb{R}$ is a $B C$ on $3^{N}$ if it satisfies the following two conditions :

$$
\begin{gathered}
\nu(\emptyset, \emptyset)=0 \\
\forall\left(A_{1}, A_{2}\right),\left(B_{1}, B_{2}\right) \in 3^{N}:\left[\left(A_{1}, A_{2}\right) \sqsubseteq\left(B_{1}, B_{2}\right) \Rightarrow \nu\left(A_{1}, A_{2}\right) \leq \nu\left(B_{1}, B_{2}\right)\right]
\end{gathered}
$$

Note that (2) is called the monotonicity condition.

In addition, a BC is said to be normalized if it satisfies :

$$
\nu(N, \emptyset)=1 \text { and } \nu(\emptyset, N)=-1
$$

$\mathrm{A} \mathrm{BC}$ is also said to be additive if the following relation holds :

$$
\forall\left(A_{1}, A_{2}\right) \in 3^{N}: \nu\left(A_{1}, A_{2}\right)=\sum_{i \in A_{1}} \nu(i, \emptyset)+\sum_{j \in A_{2}} \nu(\emptyset, j)
$$

An additive $\mathrm{BC}$ assumes that the attributes are independent from each other and this kind of $\mathrm{BC}$ boils down to linear decision models. 
In order to better formalize some of the properties of $\mathrm{BC}$, the following definition of a (bipolar) Möbius transform ${ }^{2}$ of a $\mathrm{BC}$ was proposed.

Definition 2 (Bipolar Möbius transform of a bi-capacity [10,17]) Let $\nu$ be a $B C$ on $3^{N}$. The bipolar Möbius transform of $\nu$ is a set function $b: 3^{N} \rightarrow \mathbb{R}$ defined for any $\left(A_{1}, A_{2}\right) \in 3^{N}$ by :

$$
\begin{aligned}
b\left(A_{1}, A_{2}\right) & :=\sum_{\substack{B_{1} \subseteq A_{1} \\
B_{2} \subseteq A_{2}}}(-1)^{\left|A_{1} \backslash B_{1}\right|+\left|A_{2} \backslash B_{2}\right|} \nu\left(B_{1}, B_{2}\right) \\
& =\sum_{\left(\emptyset, A_{2}\right) \sqsubseteq\left(B_{1}, B_{2}\right) \sqsubseteq\left(A_{1}, \emptyset\right)}(-1)^{\left|A_{1} \backslash B_{1}\right|+\left|A_{2} \backslash B_{2}\right|} \nu\left(B_{1}, B_{2}\right)
\end{aligned}
$$

Conversely, for any $\left(A_{1}, A_{2}\right) \in 3^{N}$, it holds that :

$$
\nu\left(A_{1}, A_{2}\right):=\sum_{\substack{B_{1} \subseteq A_{1} \\ B_{2} \subseteq A_{2}}} b\left(B_{1}, B_{2}\right) .
$$

Note that using $b,(1)$ is equivalent to :

$$
b(\emptyset, \emptyset)=0
$$

$\mathrm{BC}$ on $3^{N}$ generally require $3^{n}-1$ parameters. In order to reduce this number, $[8,9]$ and $[5]$ proposed the concept of $k$-additivity of a BC. This concept translates as follows in terms of the bipolar Möbius transform.

Proposition 1 ([17]) Given a positive integer $k<n$, a $B C \nu$ is $k$-additive if and only if the two following conditions are satisfied:

$$
\begin{gathered}
\forall\left(A_{1}, A_{2}\right) \in 3^{N}:\left|A_{1} \cup A_{2}\right|>k \Rightarrow b\left(A_{1}, A_{2}\right)=0 \\
\exists\left(A_{1}, A_{2}\right) \in 3^{N}:\left|A_{1} \cup A_{2}\right|=k \wedge b\left(A_{1}, A_{2}\right) \neq 0
\end{gathered}
$$

To avoid a heavy notation, we use the following shorthands for all $i, j \in N$, $i \neq j$ :

- $\nu_{i \mid}:=\nu(i, \emptyset), \nu_{\mid j}:=\nu(\emptyset, j), \nu_{i \mid j}:=\nu(i, j), \nu_{i j \mid}:=\nu(i j, \emptyset), \nu_{\mid i j}:=\nu(\emptyset, i j)$,

- $b_{i \mid}:=b(i, \emptyset), b_{\mid j}:=b(\emptyset, j), b_{i \mid j}:=b(i, j), b_{i j \mid}:=b(i j, \emptyset), b_{\mid i j}:=b(\emptyset, i j)$.

Whenever we use $i$ and $j$ together, it always means that they are different.

Using the above definitions, we propose the following properties of a $2 \mathrm{~A}-\mathrm{BC}$ $\nu$ and its bipolar Möbius transform $b$ :

\section{Proposition 2}

\footnotetext{
${ }^{2}$ Note that [12] was the first paper to define the Möbius transform of a BC. Their definition is different from the one given in [10]. However, there is a one-to-one correspondence between the two Möbius transform definitions. This equivalence was established in [11].
} 
1. Let $\nu$ be a 2A-BC and $b$ its bipolar Möbius transform. For any $\left(A_{1}, A_{2}\right) \in 3^{N}$ we have :

$$
\nu\left(A_{1}, A_{2}\right)=\sum_{i \in A_{1}} b_{i \mid}+\sum_{j \in A_{2}} b_{\mid j}+\sum_{\substack{i \in A_{1} \\ j \in A_{2}}} b_{i \mid j}+\sum_{\{i, j\} \subseteq A_{1}} b_{i j \mid}+\sum_{\{i, j\} \subseteq A_{2}} b_{\mid i j}
$$

2. If the coefficients $b_{i \mid}, b_{\mid j}, b_{i \mid j}, b_{i j \mid}, b_{\mid i j}$ are given for all $i, j \in N$, then the necessary and sufficient conditions to get a $2 A-B C$ generated by (10) are:

$$
\begin{aligned}
& \forall(A, B) \in 3^{N}, \forall k \in A: b_{k \mid}+\sum_{j \in B} b_{k \mid j}+\sum_{i \in A \backslash k} b_{i k \mid} \geq 0 \\
& \forall(A, B) \in 3^{N}, \forall k \in A: b_{\mid k}+\sum_{j \in B} b_{j \mid k}+\sum_{i \in A \backslash k} b_{\mid i k} \leq 0
\end{aligned}
$$

3. The inequalities (11) and (12) can be respectively reformulated in terms of the $B C \nu$ as follows :

$$
\begin{aligned}
& \forall(A, B) \in 3^{N}, \forall k \in A: \sum_{j \in B} \nu_{k \mid j}+\sum_{i \in A \backslash k} \nu_{i k \mid} \geq(|B|+|A|-2) \nu_{k \mid}+\sum_{j \in B} \nu_{\mid j}+\sum_{i \in A \backslash k} \nu_{i \mid} \\
& \forall(A, B) \in 3^{N}, \forall k \in A: \sum_{j \in B} \nu_{j \mid k}+\sum_{i \in A \backslash k} \nu_{\mid i k} \leq(|B|+|A|-2) \nu_{\mid k}+\sum_{j \in B} \nu_{j \mid}+\sum_{i \in A \backslash k} \nu_{\mid i}
\end{aligned}
$$

Proof. (Sketch of)

1. Because $\nu$ is 2 -additive, the proof of (10) is given by using the relation (6) between $\nu$ and $b$.

2. The proof of the second point is based on the expression of $\nu\left(A_{1}, A_{2}\right)$ given in (10) and on these equivalent monotonicity properties (which are easy to check) : $\forall(A, B) \in 3^{N}$ and $\forall A \subseteq A^{\prime}$,

(a) $\nu(A, B) \leq \nu\left(A^{\prime}, B\right) \Leftrightarrow\{\forall k \in A: \nu(A \backslash k, B) \leq \nu(A, B)\}$;

(b) $\nu\left(B, A^{\prime}\right) \leq \nu(B, A) \Leftrightarrow\{\forall k \in A: \nu(B, A) \leq \nu(B, A \backslash k)\}$.

3. These inequalities are obtained departing from (11) and (12) and by using the relation (6) between $\nu$ and $b$.

Hence, according to proposition 2 and (10), the computation of a $2 \mathrm{~A}-\mathrm{BC} \nu$ only requires the values of $b$ on the elements $(i, \emptyset),(\emptyset, i),(i, j),(i j, \emptyset),(\emptyset, i j)$, $\forall i, j \in N$. However, in order to satisfy the monotonicity condition given in (2) a $2 \mathrm{~A}-\mathrm{BC}$ should also satisfy the inequalities (11) and (12). Moreover, we have the following conditions in order to obtain a normalized $2 \mathrm{~A}-\mathrm{BC}$ :

$$
\nu_{N \mid}=\sum_{i \in N} b_{i \mid}+\sum_{\{i, j\} \subseteq N} b_{i j \mid}=1 \text { and } \nu_{\mid N}=\sum_{i \in N} b_{\mid i}+\sum_{\{i, j\} \subseteq N} b_{\mid i j}=-1
$$




\subsection{Bipolar Choquet integral w.r.t. a 2-additive bi-capacity}

Definition 3 (Bipolar Choquet integral (BCI) (w.r.t. a BC) [9]) Let $\nu$ be $a B C$ on $3^{N}$ and $x=\left(x_{1}, \ldots, x_{n}\right) \in \mathbb{R}^{n}$. The expression of the $B C I$ of $x$ w.r.t. $\nu$ is given by

$\mathcal{C}_{\nu}(x):=\sum_{i=1}^{n}\left|x_{\sigma(i)}\right|\left[\nu\left(N_{\sigma(i)} \cap N^{+}, N_{\sigma(i)} \cap N^{-}\right)-\nu\left(N_{\sigma(i+1)} \cap N^{+}, N_{\sigma(i+1)} \cap N^{-}\right)\right]$

where $N^{+}=\left\{i \in N \mid x_{i} \geq 0\right\}, N^{-}=N \backslash N^{+}, N_{\sigma(i)}:=\{\sigma(i), \ldots, \sigma(n)\}$ and $\sigma$ is a permutation on $N$ such that $\left|x_{\sigma(i)}\right| \leq\left|x_{\sigma(i+1)}\right| \leq \ldots \leq\left|x_{\sigma(n)}\right|$.

We also have the following equivalent expression of the BCI w.r.t. $b$, given by $[11]$ :

$$
\mathcal{C}_{b}(x)=\sum_{\left(A_{1}, A_{2}\right) \in 3^{N}} b\left(A_{1}, A_{2}\right)\left(\bigwedge_{i \in A_{1}} x_{i}^{+} \wedge \bigwedge_{j \in A_{2}} x_{j}^{-}\right)
$$

where $\left\{\begin{array}{l}x_{i}^{+}=x_{i} \text { if } x_{i}>0 \\ x_{i}^{+}=0 \text { if } x_{i} \leq 0\end{array}\right.$ and $\left\{\begin{array}{l}x_{i}^{-}=-x_{i} \quad \text { if } x_{i}<0 \\ x_{i}^{-}=0 \text { if } x_{i} \geq 0\end{array}\right.$.

Note that $\mathcal{C}_{\nu}(x)=\mathcal{C}_{b}(x)$ and the subscript is meant to clarify whether it is $\nu$ or $b$ which is used in the calculation. Besides, the BCI of $x$ w.r.t. a 2A-BC represented by $b$ reduces to :

$$
\begin{aligned}
\mathcal{C}_{b}(x)= & \sum_{i=1}^{n} b_{i \mid} x_{i}^{+}+\sum_{i=1}^{n} b_{\mid i} x_{i}^{-}+\sum_{i, j=1}^{n} b_{i \mid j}\left(x_{i}^{+} \wedge x_{j}^{-}\right) \\
& +\sum_{\{i, j\} \subseteq N} b_{i j \mid}\left(x_{i}^{+} \wedge x_{j}^{+}\right)+\sum_{\{i, j\} \subseteq N} b_{\mid i j}\left(x_{i}^{-} \wedge x_{j}^{-}\right)
\end{aligned}
$$

We have introduced the basic tools related to $2 \mathrm{~A}-\mathrm{BC}$. In the next section, we focus on the problem of identifying a $2 \mathrm{~A}-\mathrm{BC}$.

\section{Identifying a 2-additive bi-capacity}

We establish mathematical programming problems that enable the identification of a $2 \mathrm{~A}$-BC. First, we detail the type of elicitation process we are concerned with. Next, we state all the constraints that allow the representation of a $2 \mathrm{~A}$ $\mathrm{BC}$. Then, we define objective functions that reflect the quality of the identified $2 \mathrm{~A}-\mathrm{BC}$ in regard to the information provided by the DM. We end this section by illustrating the results obtained with the proposed models on a numerical example.

\subsection{Elicitation process}

In MCDM, there are two types of paradigms for elicitation processes : direct and indirect methods. In the former case, the DM is able to provide the parameters 
of his decision model directly. However, when using a BCI w.r.t. a BC, the direct method is infeasible if the number of attributes $n$ exceeds some units (typically 4 ), since a $\mathrm{BC}$ requires $3^{n}-1$ values to be set. We argued in the introduction that in order to reduce this complexity, $2 \mathrm{~A}-\mathrm{BC}$ were introduced. Nevertheless, this latter case cannot be applied in practice neither since, even if the number of parameters reduces to $2 n^{2}+1$, the complexity remains very high. Moreover, a BC is a too complex aggregation operator to ensure that a DM will understand the influence of each parameter on the final result. Even with more simple aggregation rules, it has been shown that there is no clear link between the parameters values provided by the DM and the way these values are used in the decision model [18].

Therefore, we follow the indirect paradigm. In that case, the DM does not give information about his decision model, instead, he provides information on the outputs of his decision model. In our setting, we suppose that the DM gives for some examples $x \in X^{\prime} \subseteq X$, their partial utilities for all criteria $(U(x))$ and also their overall scores $(S(x))$. We then assume, that there is no further interaction with the DM. Given the judged examples, we have to infer a decision model based on the BCI w.r.t. a 2A-BC. The estimated BCI should predict overall scores, $\mathcal{C}_{b}(x)$, that are consistent with the preference relations provided by the DM. In other words, if $S(x) \geq S\left(x^{\prime}\right)$, which means that $x$ is preferred or equivalent to $x^{\prime}$, then the inferred decision model should also satisfy $\mathcal{C}_{b}(x) \geq \mathcal{C}_{b}\left(x^{\prime}\right)$.

However, it might happen that this condition is not fulfilled for some pairs $\left(x, x^{\prime}\right)$. There are two main reasons for such situations : either the judgements provided by the DM himself are not consistent or the restriction of the decision model to 2A-BC does not allow fitting the DM preferences correctly. In MCDM, inconsistencies are usually treated in an interaction loop with the DM. It is assumed that the DM preferences can change in order to fix these incoherences when they are encountered. In our setting, the interaction loop is not permitted. Consequently, in order to cope with incoherences, we propose two versions of our models : the first one does not deal with inconsistencies and thus will return that the problem is infeasible if any incoherence is encountered whereas the second one allows inconsistencies and attempts to infer a model that minimizes errors due to such situations as much as possible.

\subsection{Mathematical programming problems}

We propose two types of optimization problems to identify a $2 \mathrm{~A}-\mathrm{BC}$ in the context we have described in the previous paragraph. We base our work on some of the elicitation methods detailed in [14] in the case of unipolar Choquet integral. Before introducing the objective functions of our optimization problems, we start by enumerating the different sets of constraints that need to be satisfied.

We represent the unknown $2 \mathrm{~A}-\mathrm{BC} \nu$, via its associated bipolar Möbius transform $b$. There are two reasons for this. Firstly, equations (5) and (6) state that it is equivalent to work with either $\nu$ or $b$. Secondly, since we restrict the BC to be 2 -additive and since this property, given in (8) and (9), is defined in terms of 
$b$, it is thus necessary to use the latter representation in our optimization problems. However, we do not take (9) into account in our set of constraints. This equation ensures that $\nu$ is exactly 2 -additive and by discarding it, we explicitly allow $b$ to be either 2 -additive or simply additive. To summarize this first set of constraints, we need to integrate the following relations in our optimization problems in order to have a normalized 2A-BC in terms of $b:(7)$, (8) with $k=2$, (11), (12) and (13).

Next, we have to take into account the preference relations provided by the DM on the subset of examples $X^{\prime}$. If $S(x) \geq S\left(x^{\prime}\right)$ then the BCI should be in concordance with this inequality. Accordingly, we have the following second set of constraints :

$$
\forall x, x^{\prime} \in X^{\prime}, x \neq x^{\prime}: S(x)-S\left(x^{\prime}\right) \geq 0 \Rightarrow \mathcal{C}_{b}(x)-\mathcal{C}_{b}\left(x^{\prime}\right) \geq \delta_{c}
$$

where $\delta_{c}$ is a non-negative indifference threshold which is a parameter of the model. Note that this set of constraints does not allow incoherences. Indeed, the inferred $2 \mathrm{~A}$-BC $b$ could not be flexible enough to satisfy $\mathcal{C}_{b}(x)-\mathcal{C}_{b}\left(x^{\prime}\right) \geq \delta_{c}$ for some pairs $\left(x, x^{\prime}\right)$. In that case the optimization problem is infeasible.

As discussed previously, in order to overcome this drawback, we transform the previous constraints as follows :

$$
\forall x, x^{\prime} \in X^{\prime}, x \neq x^{\prime}: S(x)-S\left(x^{\prime}\right) \geq 0 \Rightarrow \mathcal{C}_{b}(x)-\mathcal{C}_{b}\left(x^{\prime}\right) \geq \delta_{c}-\xi_{x x^{\prime}}
$$

where $\xi_{x x^{\prime}}$ are non-negative slack variables which allow inconsistencies. However, we want $\xi_{x x^{\prime}}$ to be has low as possible and thus there should be a term in the objective function seeking to minimize $\sum_{x, x^{\prime}: S(x) \geq S\left(x^{\prime}\right)} \xi_{x x^{\prime}}$. Note that when the latter term is null, it means that the inferred model does not produce any incoherence. On the contrary, if for some pairs $\left(x, x^{\prime}\right), \xi_{x x^{\prime}}>\delta_{c}$ then the optimal solution has not been able to satisfy the preference relations on these pairs.

The third set of constraints is related to the computation of the BCI. Indeed, in (17) or (18), we need to calculate $\mathcal{C}_{b}(x)$ for each $x \in X^{\prime}$. As a consequence, we need to add the constraints provided by (16) in our models. Note that despite the fact that the latter equations involve the minimum function, we can pre-compute the terms $\left(x_{i}^{ \pm} \wedge x_{j}^{ \pm}\right)$since they are parameters of the models. Consequently, the constraints (16) are linear equations.

The fourth set of constraints is optional. It simply consists in adding upper and lower bounds for the BCI values :

$$
\forall x \in X^{\prime} \quad: l b \leq \mathcal{C}_{b}(x) \leq u b
$$

where $l b$ and $u b$ are two real parameters.

After having introduced the constraints, we now focus on the different objective functions and the resulting optimization problems.

We propose two kinds of optimization models. In the first approach, we extend the maximum split method introduced in [19]. This model assumes the following constraints in place of (17) :

$$
\forall x, x^{\prime} \in X^{\prime}, x \neq x^{\prime}: S(x)-S\left(x^{\prime}\right) \geq 0 \Rightarrow \mathcal{C}_{b}(x)-\mathcal{C}_{b}\left(x^{\prime}\right) \geq \delta_{c}+\varepsilon
$$


where $\varepsilon$ is a variable of the problem unlike $\delta_{c}$ which is a parameter. The objective function consists in maximizing $\varepsilon$. In other words, we want to maximize the difference (split) $\mathcal{C}_{\nu}(x)-\mathcal{C}_{\nu}\left(x^{\prime}\right)$ for any $x \neq x^{\prime} \in X^{\prime}$ such that $S(x) \geq S\left(x^{\prime}\right)$. We refer to the following optimization problem as the split method : max $\varepsilon$ subject to $(7)$, (8) with $k=2,(11),(12),(13),(16),(19)$ and (20).

However, the split model does not address incoherences. Hence, as explained previously, we propose the split flex approach which uses the following third set of constraints instead of (20):

$$
\forall x, x^{\prime} \in X^{\prime}, x \neq x^{\prime}: S(x)-S\left(x^{\prime}\right) \geq 0 \Rightarrow \mathcal{C}_{b}(x)-\mathcal{C}_{b}\left(x^{\prime}\right) \geq \delta_{c}+\varepsilon-\xi_{x x^{\prime}}
$$

More formally, the split flex model is defined by : $\max \varepsilon-\sum_{x, x^{\prime}: S(x) \geq S\left(x^{\prime}\right)} \xi_{x x^{\prime}}$ subject to (7), (8) with $k=2,(11),(12),(13),(16),(19)$ and (21). Note that the split and split flex optimization problems have linear objective functions and linear constraints. Therefore, there are linear programs.

We now present the second type of model for $2 \mathrm{~A}-\mathrm{BC}$ identification. This approach is a regression-like method and yields to quadratic programs. We propose to minimize the sum of square errors between $S$ and $\mathcal{C}_{b}$ which results in the following objective function : $\min \sum_{x, x^{\prime} \in X^{\prime}}\left(S(x)-\mathcal{C}_{b}(x)\right)^{2}$. Accordingly, we named rss (for Residual Sum of Square) the following problem : $\min \sum_{x, x^{\prime} \in X^{\prime}}\left(S(x)-\mathcal{C}_{b}(x)\right)^{2}$ subject to $(7),(8)$ with $k=2,(11),(12),(13),(16)$ and (17). Similarly to split, the rss method does not permit incoherences.

As a consequence, we introduce a flexible version of $r s s$ that we call rss flex and which is given by $: \min \sum_{x, x^{\prime} \in X^{\prime}}\left(S(x)-\mathcal{C}_{b}(x)\right)^{2}+\sum_{x, x^{\prime}: S(x) \geq S\left(x^{\prime}\right)} \xi_{x x^{\prime}}$ subject to $(7)$, (8) with $k=2,(11),(12),(13),(16)$ and (18).

\subsection{An illustrative example}

We applied the four different mathematical programming problems defined previously on a numerical example taken from [14]. It concerns the grades (utilities) obtained by 7 students (alternatives) for $n=5$ subjects (attributes) : statistics $(\mathrm{S})$, probability $(\mathrm{P})$, economics $(\mathrm{E})$, management $(\mathrm{M})$, and English (En). The grades globally belong to $[0,20]$ but in this example, the scores only vary in $[11,18]$. In our perspective, we transformed them in order to have a bipolar scale by simply applying a translation of -14 to the original scores. Therefore, in this bipolar scale, the scores belong to $[-3,4]$. Suppose that a student is delivered his diploma with honors providing that his overall grade is greater or equal to 14. Hence, the translated scores in the bipolar scale allow us to deal with the decision problem of delivering honors as follows : the student is attributed the honors if and only if his overall grade in the bipolar scale is non-negative.

The performance table is given in Table 1 (a). In Table 1 (b), the first column $S$ corresponds to the (translated) overall grades as given in [14]. Then, in the subsequent columns of Table 1 (b), we show the different estimated scores. Note that for all models we set $\delta_{c}=0.5, l b=-3$ and $u b=4$. For split and split flex, even if the inferred overall scores are not the same, the two solutions are actually equivalent since they give the same objective function value (the problem is not 


\begin{tabular}{|c|c|c|c|c|c|c|c|c|c|c|c|}
\hline Student & S P E M En & $S$ & split & $\begin{array}{l}\text { split } \\
\text { flex }\end{array}$ & $r s s$ & $\begin{array}{l}\text { rss } \\
\text { flex }\end{array}$ & $S^{\prime}$ & split & $\begin{array}{l}\text { split } \\
\text { flex }\end{array}$ & $r s s$ & $\begin{array}{l}\text { rss } \\
\text { flex }\end{array}$ \\
\hline$a$ & $4-3-3-3 \quad 4$ & 1 & 1.68 & 1.02 & 1 & 1 & 1 & . & 0.22 & $\overline{.}$ & 1.12 \\
\hline$b$ & $\begin{array}{lllll}4 & -3 & 4 & -3 & -3\end{array}$ & 0.5 & 1.04 & 0.38 & 0.5 & 0.5 & 0.5 & . & -0.28 & . & 0.62 \\
\hline$c$ & $\begin{array}{lllll}-3 & -3 & 4 & -3 & 4\end{array}$ & 0 & 0.41 & -0.25 & 0 & 0 & 0 & . & -0.78 & . & 0.12 \\
\hline$d$ & $\begin{array}{lllll}4 & 4 & -3 & -3 & -3\end{array}$ & -0.5 & -0.23 & -0.89 & -0.5 & -0.5 & -0.5 & . & -1.28 & . & -0.5 \\
\hline$e$ & $\begin{array}{lllll}-3 & -3 & 4 & 4 & -3\end{array}$ & -1 & -0.86 & -1.53 & -1 & -1 & -1 & . & -1.78 & . & -1 \\
\hline$f$ & $\begin{array}{lllll}-3 & -3 & 4 & -3 & -3\end{array}$ & -1.5 & -1.5 & -2.16 & -1.5 & -1.5 & -1.5 & . & -2.28 & . & -1.5 \\
\hline$g$ & $\begin{array}{lllll}-3 & -3 & -3 & -3 & 4\end{array}$ & -2 & -2.14 & -2.8 & -2 & -2 & 0.5 & . & -0.78 & . & 0.12 \\
\hline
\end{tabular}

Table 1. (a) Performance table; (b) Results obtained with the original (translated) overall score $S$; (c) Results obtained with the modified overall score $S^{\prime}$ that presents inconsistencies.

strictly concave). However, in terms of decisions, the sign of the overall grade for $c$ is not the same for the two models. This example exhibits some limits of this type of model. Besides, we precise that in split flex outputs, all slack variables $\xi_{x x^{\prime}}$ are null which means that there is no inconsistency. Regarding rss and rss flex, we obtain a null objective function value and in the latter case, the slack variables are also all null as expected.

To illustrate the case with incoherences, we modified the overall score $S$ into $S^{\prime}$. We simply change the global grade of $g$ from -2 to 0.5 . This new score is an example of inconsistent preferences provided by the DM since his decision model is not monotonic in that case. Indeed, if we compare the profiles of $c$ and $g$ in regard to their overall score $S^{\prime}(c)$ and $S^{\prime}(g)$, we can observe that $g$ is preferred to $c$ while the scores distribution of the former student is Pareto dominated by the latter one. This situation is not consistent with a rational decision. Table 1 (c) presents the estimated BCI. As expected, split and rss returned an infeasible problem. On the contrary, split flex and rss flex provide interesting results. In both cases, we precise that $\xi_{g c}=0.5$ while the other slack variables are null.

We present in Table 2 the elicited bipolar Möbius transform $b$ for each optimization problem when $S$ is the targeted overall grades vector. In Table 3 , it is the estimated $b$ for the split flex and rss flex methods when $S^{\prime}$ is put in place of $S$, which are shown. Notice that an empty cell in these two tables means that the solver returned a null value for the corresponding elements.

We show the bipolar Möbius transform $b$ and not the 2A-BC $\nu$ because the latter set function requires $3^{5}-1=242$ non-null values which represents a too large table. In contrast, because $\nu$ is 2 -additive, we have at most $2 \times 5^{2}+1=51$ values for its related bipolar Möbius transform $b$.

The utility of presenting Tables 2 and 3 , is that they allow one to check that the constraints (7), (8) with $k=2,(11),(12),(13)$ are indeed satisfied. We thus obtain a 2A-BC. However, it is difficult to interpret the bipolar Möbius transform $b$ with regard to the underlying elicited preference model. Moreover, from this illustrative example, it is not straightforward to understand the impact of taking into account inconsistencies either when we compare the regular and the flex 


\begin{tabular}{|c|c|c|c|c|c|c|c|c|}
\hline & \multicolumn{2}{|c|}{ split } & \multicolumn{2}{|c|}{ split flex } & \multicolumn{2}{|c|}{$r s s$} & \multicolumn{2}{|c|}{ rss flex } \\
\hline$A_{1} \quad A_{2}$ & $b_{A_{1} \mid A_{2}}$ & $b_{A_{2} \mid A_{1}}$ & $b_{A_{1} \mid A_{2}}$ & $b_{A_{2} \mid A_{1}}$ & $b_{A_{1} \mid A_{2}}$ & $b_{A_{2} \mid A_{1}}$ & $b_{A_{1} \mid A_{2}}$ & $b_{A_{2} \mid A_{1}}$ \\
\hline$\emptyset \quad \emptyset$ & & & & & & & & \\
\hline$\emptyset \quad \mathrm{S}$ & & 0.26 & -0.145 & & -0.039 & 0.077 & & 0.056 \\
\hline $\mathrm{P}$ & & & & & -0.077 & 0.038 & -0.056 & \\
\hline $\mathrm{E}$ & & & & 0.145 & -0.038 & 0.077 & & 0.056 \\
\hline $\mathrm{M}$ & & & & & -0.08 & 0.038 & & \\
\hline En & & & -0.066 & & -0.039 & 0.008 & -0.056 & \\
\hline$\emptyset \quad$ SP & & & -0.56 & & -0.33 & & -0.24 & \\
\hline $\mathrm{SE}$ & -0.076 & & & 0.11 & -0.004 & 0.11 & & 0.19 \\
\hline $\mathrm{SM}$ & -0.21 & & & & & -0.008 & & \\
\hline$\emptyset \quad$ SEn & -0.29 & 0.48 & & 0.43 & 0.039 & 0.47 & & 0.53 \\
\hline$\emptyset \quad \mathrm{PE}$ & & & & & -0.29 & & -0.43 & \\
\hline$\emptyset \quad \mathrm{PM}$ & & & & & & -0.03 & & \\
\hline$\emptyset \quad$ PEn & & & & & & -0.008 & & \\
\hline$\emptyset \quad \mathrm{EM}$ & -0.42 & & -0.23 & & 0.004 & & & \\
\hline$\emptyset \quad \mathrm{EEn}$ & & 0.26 & & 0.32 & -0.14 & 0.23 & -0.056 & 0.17 \\
\hline$\emptyset$ MEn & & & & & & & -0.17 & \\
\hline $\begin{array}{ll}\mathrm{S} & \mathrm{P}\end{array}$ & & & & & & & & \\
\hline $\mathrm{E}$ & & & & & -0.07 & -0.039 & & -0.056 \\
\hline M & & & & 0.145 & 0.004 & & -0.02 & \\
\hline En & & & & & 0.039 & 0.039 & 0.056 & \\
\hline $\mathrm{E}$ & & & & & & & & \\
\hline M & & & & & & 0.039 & & \\
\hline En & & & & & & 0.038 & & 0.056 \\
\hline M & & & & & 0.004 & & & \\
\hline En & & & -0.145 & & -0.039 & 0.034 & & \\
\hline En & & & 0.066 & & & 0.073 & & \\
\hline
\end{tabular}

Table 2. Values of the elicited bipolar Möbius transform $b$ when $S$ is the overall score.

versions of split and rss models in Table 2, or when we look at the obtained $b$ for $S$ in Table 2 and the one estimated for $S^{\prime}$ in Table 3. Accordingly, further experiments should be undertaken in order to have a better understanding of the behaviors of the elicited $\nu$ and $b$ for each proposed optimization problem but such an experimental study is out of the scope of this paper.

\section{Relationships with machine learning}

The preference elicitation problem have many common points with supervised learning problems (SL) in statistical machine learning (ML). In the latter field, we are given a training set which consists of items described in a feature space and each element also comes with a value in regard to a target variable. In ML, the goal of SL is to infer from the training set a mapping from the feature space to the target variable. This description if similar to the preference elicitation setting we have described previously : the set of examples with their profiles 


\begin{tabular}{|cc|cc|cc|}
\hline & & \multicolumn{2}{|c|}{ split flex } & \multicolumn{2}{|c|}{ rss flex } \\
\hline$A_{1}$ & $A_{2}$ & $b_{A_{1} \mid A_{2}}$ & $b_{A_{2} \mid A_{1}}$ & $b_{A_{1} \mid A_{2}}$ & $b_{A_{2} \mid A_{1}}$ \\
\hline$\emptyset$ & $\mathrm{S}$ & & & -0.077 & 0.47 \\
$\emptyset$ & $\mathrm{P}$ & -0.19 & & -0.53 & 0.077 \\
$\emptyset$ & $\mathrm{E}$ & & & -0.077 & 0.023 \\
$\emptyset$ & $\mathrm{M}$ & & 0.031 & -0.14 & 0.077 \\
$\emptyset$ & $\mathrm{En}$ & -0.031 & & & 0.19 \\
\hline$\emptyset$ & $\mathrm{SP}$ & -0.26 & & 0.077 & 0.09 \\
$\emptyset$ & $\mathrm{SE}$ & & 0.19 & & 0.263 \\
$\emptyset$ & $\mathrm{SM}$ & & 0.73 & & \\
$\emptyset$ & $\mathrm{SEn}$ & -0.16 & 0.055 & & -0.018 \\
$\emptyset$ & $\mathrm{PE}$ & & & & \\
$\emptyset$ & $\mathrm{PM}$ & & & 0.14 & \\
$\emptyset$ & $\mathrm{PEn}$ & 0.031 & & & -0.077 \\
$\emptyset$ & $\mathrm{EM}$ & & & & \\
$\emptyset$ & $\mathrm{EEn}$ & -0.24 & & -0.39 & -0.023 \\
$\emptyset$ & $\mathrm{MEn}$ & -0.16 & & & -0.077 \\
\hline $\mathrm{S}$ & $\mathrm{P}$ & & & 0.06 & \\
$\mathrm{~S}$ & $\mathrm{E}$ & & & & \\
$\mathrm{S}$ & $\mathrm{M}$ & & -0.031 & -0.33 & \\
$\mathrm{~S}$ & $\mathrm{En}$ & & & & \\
$\mathrm{P}$ & $\mathrm{E}$ & & & \multicolumn{2}{|c}{} \\
$\mathrm{P}$ & $\mathrm{M}$ & & & & 0.14 \\
$\mathrm{P}$ & $\mathrm{En}$ & & 0.19 & -0.077 & 0.31 \\
$\mathrm{E}$ & $\mathrm{M}$ & & & & \\
$\mathrm{E}$ & $\mathrm{En}$ & & & & 0.077 \\
$\mathrm{M}$ & $\mathrm{En}$ & & & -0.077 & \\
\hline
\end{tabular}

Table 3. Values of the elicited bipolar Möbius transform $b$ when $S^{\prime}$ is the overall score with incoherences.

$U(x)$ and their overall scores $S(x)$ are the equivalent of the training set in ML and identifying the parameters of the decision model in order to reproduce the preference relations given by the DM is the same as inferring a mapping from a feature space $(X)$ and a target variable $(S)$. Despite these straightforward similarities, the roots of these two domains are distinct, and it is only recently, that there has been a growing interest in applying concepts or/and techniques developed in MCDM to ML problems and vice-versa [16].

To contribute in that direction, we discuss the relationships between our work and some concepts defined in ML.

Firstly, in MCDM, incoherences are not generally allowed from the DM viewpoint who has to fix them during the course of the elicitation procedure. In ML, on the contrary, such situations are typically observed in real-world applications. Thus, the models split and rss are typical of MCDM whereas split flex and rss flex are closer to SL problems. 
Secondly, it is noteworthy that the restriction to $2 \mathrm{~A}-\mathrm{BC}$ can be interpreted as an explicit regularization of the decision model based on the BCI. Indeed, in SL, regularization is a concept that aims at dealing with the bias-variance trade-off of predictive models. Typically, in order to avoid an over-fitting effect, we allow the estimated predictive model to make more errors on the training set (bias) but in return we want it to be less variable (variance). The goal of regularization is to enhance the ability of the inferred model to predict correctly the overall score of observed examples (the training set) but also and in particular the global score of unseen examples (the test set). In our case, 2A-BC are less flexible than unconstrained $\mathrm{BC}$ and this could lead to more inconsistencies as explained beforehand. However, the 2-additivity property makes the $\mathrm{BC}$ less complex and we intuitively expect $2 \mathrm{~A}-\mathrm{BC}$ to be less variable than unconstrained $\mathrm{BC}$.

Thirdly, we stated that the constraint (9) ensuring that $\nu$ is exactly 2 -additive was not part of our set of constraints. In that case $\nu$ could thus be either 2additive or simply additive. We can make the correspondence between this approach and the Occam's Razor principle often used in ML. This concept states that one should prefer simpler models than more complex ones because they allow a better understanding of the phenomenon under study. In our case, we can transpose this statement as follows : if an additive BC fits better the DM judgements than a $2 \mathrm{~A}-\mathrm{BC}$ then we should go for the former one. By discarding (9) from our constraints we make the latter statement possible. However, if it happens that a $2 \mathrm{~A}-\mathrm{BC}$ and an additive $\mathrm{BC}$ yield to the same optimal objective function value then it is not guaranteed that the optimization solver will provide the simplest solution.

Finally, in order to make the models split and rss more flexible regarding inconsistencies, we have proposed to integrate slack variables in the constraints and in the objective function as well. Our approach is inspired from the Support Vector Machine (SVM) method developed in SL in order to deal with non linearly separable cases in binary classification [20]. However, it is noteworthy that the UTA (UTilités Additives) framework is a MCDM methodology that also addresses inconsistencies by integrating overestimation and underestimation error variables in the elicitation model (see for example [21]). This approach is similar to adding slack variables.

\section{Conclusion}

We have proposed optimization problems that allow the identification of the parameters of a $2 \mathrm{~A}-\mathrm{BC}$. The decision model is inferred from examples that the DM evaluated both regarding their partial utilities and their global scores. We have considered the traditional preference elicitation setting where no inconsistency is allowed. But, we have also extended the models to the more flexible case where we have to cope with such incoherences. In this context, we have emphasized the relationships between our approaches and concepts in ML.

In our future work, we intend to integrate other kinds of information provided by the DM such as the importance of criteria and the interactions between them. 
Moreover, as pointed out previously, additional experiments should be conducted in order to better characterize the elicited bipolar Möbius transform $b$ and its associated 2A-BC $\nu$, we obtain for each type of mathematical program.

As regard to our ongoing work, we are investigating optimization problems for preferences learning that integrate other concepts developed in the ML literature. We are currently working on objective functions that involve a penalty term that favors sparse BC. Such an approach adds a trade-off between the accuracy of the model and its simplicity (Occam's Razor principle). The expected advantage is to elicit preference models that are easier to interpret and such a feature is of great importance in MCDM.

\section{References}

1. Choquet, G.: Theory of capacities. Annales de l'institut Fourier 5 (1954) 131-295

2. Grabisch, M.: k-order additive discrete fuzzy measures and their representation. Fuzzy Sets and Systems 92(2) (1997) $167-189$

3. Grabisch, M., Labreuche, C.: Fuzzy measures and integrals in MCDA. In: Multiple Criteria Decision Analysis: State of the Art Surveys. Volume 78 of Int. Series in Op. Res. \& Manag. Sci. Springer New York (2005) 563-604

4. Meyer, P., Pirlot, M.: On the expressiveness of the additive value function and the choquet integral models. In: DA2PL'2012 From Multiple Criteria Decision Aid to Preference Learning. (2012)

5. Grabisch, M., Labreuche, C.: A decade of application of the Choquet and Sugeno integrals in multi-criteria decision aid. 4OR 6(1) (2008) 1-44

6. Grabisch, M., Labreuche, C.: Bi-capacities for decision making on bipolar scales. In: Proc. of the EUROFUSE 02 Workshop on Information Systems. (2002) 185-190

7. Greco, S., Matarazzo, B., Slowinski, R.: Bipolar Sugeno and Choquet integrals. In: Proceedings of the EUROFUSE 02 Workshop on Information Systems. (2002)

8. Grabisch, M., Labreuche, C.: Bi-capacities-I: definition, Möbius transform and interaction. Fuzzy Sets and Systems 151(2) (2005) $211-236$

9. Grabisch, M., Labreuche, C.: Bi-capacities-II: the Choquet integral. Fuzzy Sets and Systems 151(2) (2005) $237-259$

10. Fujimoto, K.: New characterizations of $k$-additivity and $k$-monotonicity of bicapacities. In: Joint 2nd Int. Conf. on Soft Computing and Intelligent Systems and 5th International Symposium on Advanced Intelligent Systems. (2004)

11. Fujimoto, K., Murofushi, T.: Some characterizations of $k$-monotonicity through the bipolar möbius transform in bi-capacities. JACIII 9(5) (2005) 484-495

12. Grabisch, M., Labreuche, C.: The choquet integral for 2-additive bi-capacities. In: EUSFLAT Conf. (2003) 300-303

13. Jacquet-Lagreze, E., Siskos, J.: Assessing a set of additive utility functions for multicriteria decision-making, the UTA method. European Journal of Operational Research 10(2) (1982) $151-164$

14. Grabisch, M., Kojadinovic, I., Meyer, P.: A review of methods for capacity identification in Choquet integral based multi-attribute utility theory: Applications of the kappalab R package. EJOR 186(2) (2008) 766 - 785

15. Mayag, B., Rolland, A., Ah-Pine, J.: Elicitation of a 2-additive bi-capacity through cardinal information on trinary actions. In: IPMU (4). (2012) 238-247

16. Waegeman, W., Baets, B.D., Boullart, L.: Kernel-based learning methods for preference aggregation. 4OR 7(2) (2009) 169-189 
17. Fujimoto, K., Murofushi, T., Sugeno, M.: k-additivity and c-decomposability of bi-capacities and its integral. Fuzzy Sets Syst. 158(15) (August 2007) 1698-1712

18. Bouyssou, D., Marchant, T., Pirlot, M., Tsoukias, A., Vincke, P.: Evaluation and Decision models with multiple criteria: stepping stones for the analyst. Volume 86 of Int. Series in Op. Res. \& Manag. Sci. Springer (2006)

19. Marichal, J.L., Roubens, M.: Determination of weights of interacting criteria from a reference set. EJOR 124(3) (2000) 641 - 650

20. Cortes, C., Vapnik, V.: Support-vector networks. Mach. Learn. 20(3) (September 1995) 273-297

21. Siskos, Y., Grigoroudis, E., Matsatsinis, N.: UTA methods. In: Multiple Criteria Decision Analysis: State of the Art Surveys. Volume 78 of International Series in Operations Research \& Management Science. Springer New York (2005) 297-334 